\title{
Molecular Structure of I sotactic Polypropylene Formed from Homogeneous Solution. Gelation and Crystallization
}

\author{
Takahiko NAKAOKI and Yoritsugu INAJI \\ Faculty of Science and Technology, Ryukoku University, Seta, Otsu 520-2194, Japan
}

(Received March 14, 2002; Accepted May 17, 2002)

\begin{abstract}
The molecular structure and morphology grown from isotactic polypropylene (iPP)/o-dichlorobenzene solution were investigated by thermal analysis and high-resolution solid-state ${ }^{13} \mathrm{C}$ NMR. The morphology varied depending on incubated temperature. A gelation occurred below $70^{\circ} \mathrm{C}$, while the crystallization took place over $80^{\circ} \mathrm{C}$. The crystal obtained was identified with the $\alpha$-form, which is the most stable crystalline modification of iPP. In CP/MAS ${ }^{13} \mathrm{C}$ NMR spectrum, the chemical shift of methylene, methine, and methyl carbons provided almost the same shift for both the gel and $\alpha$-form, but the characteristic doublet for methylene and methyl signals of the gel was different in intensity ratio with the $\alpha$-form. These results indicate that the gel-forming crystal is identified with $\alpha_{1}$-form, being the disordered $\alpha$-form. Indeed, the lower melting temperature and smaller melting enthalpy than the crystal were observed by thermal analysis. This result connects to the incompleteness of crystal formed in the gel.

KEY WORDS Isotactic Polypropylene / Gelation / Crystallization / Morphology / Thermal Analysis / Solid-State ${ }^{13} \mathrm{C}$ Nuclear Magnetic Resonance (NMR) /
\end{abstract}

Isotactic polypropylene (iPP) shows polymorphs as a function of crystallization condition. As for the molecular conformation, all crystal modifications take a regular trans-gauche $(\operatorname{tg})$ conformation with $3 / 1$ helix. The most thermodynamically stable form takes a monoclinic structure called $\alpha$-form. ${ }^{1}$ The pseudohexagonal $\beta$-form is obtained by high cooling rate or by adding specific nucleating agents..$^{2-8}$ The orthorhombic $\gamma$-form has unique chain structure which is featured by nonparallel chain axes to one another. ${ }^{8-12}$ This form can be obtained by crystallization for low molecular weight iPP or by dealing at high pressure. There is also a mesomorphic structure which can be obtained by quenching from the melt. The investigation of this form has been performed by lots of researchers, who proposed the interpretations such as smectic phase, ${ }^{2}$ paracrystal, ${ }^{13,14}$ microcrystallite, ${ }^{15-18}$ and so on. The recent study seems to reach the conclusion that the ordered helical chain distributes without longitudinal order, however the crystalline unit is close to the structure of the monoclinic $\alpha$-form. ${ }^{19}$

In order to investigate these crystalline modifications, high-resolution solid-state ${ }^{13} \mathrm{C}$ NMR is a powerful tool. First Bunn et al. reported the characteristic doublet with $2: 1$ intensity ratio for methylene and methyl signals in cross polarization (CP) / magic angle spinning (MAS) ${ }^{13} \mathrm{C}$ NMR. ${ }^{20}$ After that, lots of investigations using high-resolution solid-state ${ }^{13} \mathrm{C}$ NMR were carried out. ${ }^{7,16,17,21}$ Caldas et al. investigated the relationship between the crystalline form and the intensity ratio of doublet, and established the origin of different intensity ratio for the lower crystalline order form $\left(\alpha_{1}\right)$ with $\mathrm{C} 2 / \mathrm{c}$ and the higher crystalline order form $\left(\alpha_{2}\right)$ with $\mathrm{P} 2{ }_{1} / \mathrm{c}$ space group. ${ }^{16-18}$ Two different environments giving doublet exist in the crystal cell depending on the arrangement of left- and right-handed helices at the interlayer region.

A gelation as well as crystallization is another recent topic. Generally the gelation accompanies the formation of three dimensional network structure consisted of crystalline as a cross-linking point. It is reported that some experimental results of the gelforming crystal provide small differences with bulkcrystallized structure. For example, infrared spectrum of isotactic polystyrene (iPS) gel has the characteristic peaks in $900-1000 \mathrm{~cm}^{-1}$ region, although other peaks are identified with $\alpha$-iPS crystal. $^{22}$ Another example is syndiotactic polypropylene (sPP) gel which is featured by the three distinct methylene signals observed in high-resolution solid-state ${ }^{13} \mathrm{C}$ NMR. ${ }^{23,24}$ This characteristic split was only observed for the gel. In case of iPP gel, we revealed that $\mathrm{CP} / \mathrm{MAS}{ }^{13} \mathrm{C}$ NMR spectrum of the gel quenched at $0^{\circ} \mathrm{C}$ is characterized by the doublet with $1: 2$ intensity ratio from downfield for methylene signal, which is inverse intensity ratio with the $\alpha$ form. ${ }^{25}$

In this paper, we concentrated to elucidate the solution grown structure for $\mathrm{iPP} / o$-dichlorobenzene system. The molecular structure was investigated as a function of temperature quenched from the homogeneous solution by using thermal analysis and high-resolution solid-state ${ }^{13} \mathrm{C}$ NMR spectroscopy. 


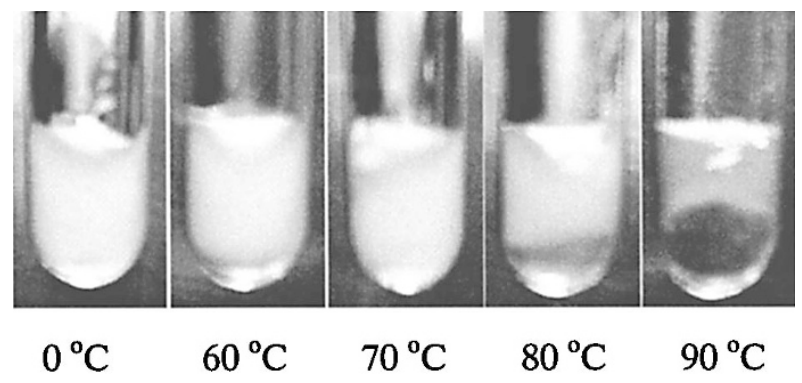

Figure 1. Photographs for iPP/o-dichlorobenzene incubated at given temperature. Below $70^{\circ} \mathrm{C}$, a homogeneous gel was formed. Above $80^{\circ} \mathrm{C}$, the system separated to two phases; the upper phase was white precipitation consisting of the $\alpha$-form, while the lower phase was viscous liquid.

\section{EXPERIMENTAL}

A sample of iPP was purchased from Showa Denko Co. Ltd. It was purified by soxlet extraction with $n$ heptane. The meso triad was estimated to be $98.5 \%$ by ${ }^{13} \mathrm{C}$ NMR. The solution grown samples were prepared as follows. Both iPP and $o$-dichlorobenzene (13 wt\%) were placed in an ampoule and degassed in vacuo and sealed. The solution was homogeneously solved at $170^{\circ} \mathrm{C}$, and then quenched into thermostat kept at desired temperature.

Thermal analysis was performed by a Rigaku TAS 200 differential scanning calorimeter (DSC). The heating rate was $10^{\circ} \mathrm{C} \mathrm{min}^{-1}$. The measurements were carried out 3 times and then the average values were calculated. Calibration of the instrument for temperature and enthalpy was accomplished by indium.

High-resolution solid-state ${ }^{13} \mathrm{C}$ NMR spectra were taken with a Bruker MSL 200 spectrometer at a field strength of 4.7 Tesla. The ${ }^{13} \mathrm{C}$ chemical shifts were obtained with respect to the signal of tetramethylsilane (TMS). The magic angle spinning of $3 \mathrm{kHz}$ was achieved by the double bearing system. CP/MAS experiments were performed applying a recycle delay of $5 \mathrm{~s}$ and a contact time of $1 \mathrm{~ms}$. The measurements were carried out at room temperature. To avoid the broadening of signals, we did not use window function except the Lorentz-Gauss conversion analysis.

\section{RESULTS AND DISCUSSION}

Thermodynamical stability of two-phase system of polymer and solvent basically depends on temperature and concentration. In order to simplify the experimental result, the concentration was fixed at $13 \mathrm{wt} \%$ in this study, and then the temperature quenched from the homogeneous solution was changed. Figure 1 shows the photograph of samples kept at given temperature.

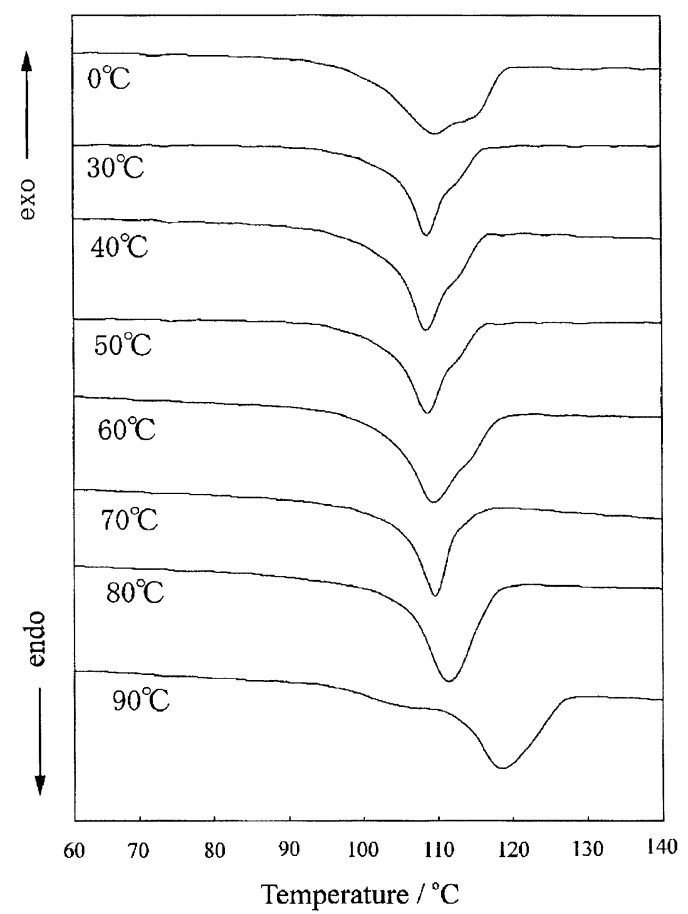

Figure 2. DSC thermograms for the samples incubated at given temperature.

Below $70^{\circ} \mathrm{C}$, the gelation took place, while the system separated to two phases above $80^{\circ} \mathrm{C}$. The bottom phase was clear solution with high viscosity, while upper phase was a fibrillar-like precipitation. In this system, the density of $o$-dichlorobenzene $\left(1.3 \mathrm{~g} \mathrm{~cm}^{-3}\right)$ is larger than that of the polymer so that the precipitation locates at upper phase. The aggregation of polymer would be strongly accelerated at high temperature, since the smaller surface energy makes the system stabilize. As a result, the white solid crystal was precipitated from the solution. In general, the gelation for crystalline polymer like iPP accompanies the formation of crystal as a cross-linking point. The polarized microscopy revealed that the spherulites were observed in the iPP gel. ${ }^{26}$ Since there is no phase separation below $70^{\circ} \mathrm{C}$, the molecular chains make entanglement with neighboring chains. Due to the crystallization between chains, the three dimensional network structure was formed in the system. These results show that the aggregation process for gelation is regarded as the same, namely crystallization occurred, but the molecular morphology is different between the gel and crystal.

\section{Thermal Behavior}

Figure 2 shows DSC profiles depending on incubated temperature. The endothermic peak corresponds to the melting for the gel or the crystal. The gel formed at $0^{\circ} \mathrm{C}$ provided the broad peak, but with increasing incubated temperature the profile became sharp. The peak top temperature was plotted against incubated temper- 


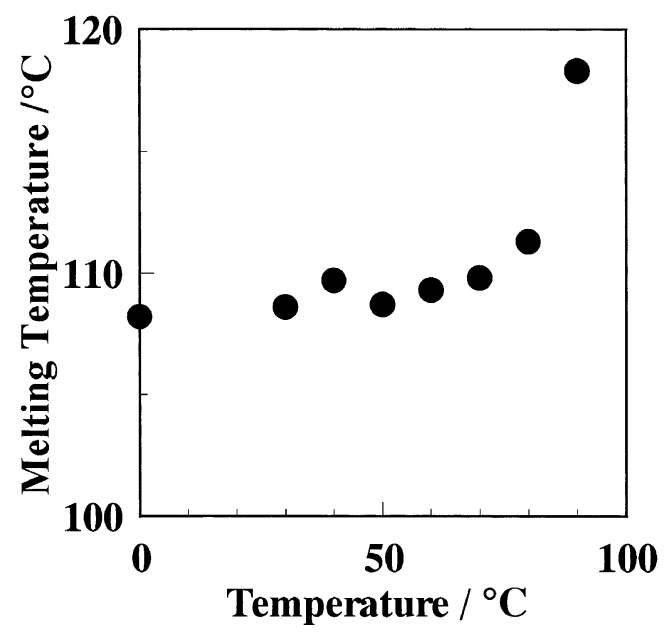

Figure 3. Melting temperature for the gel and the crystal depending on incubated temperature.

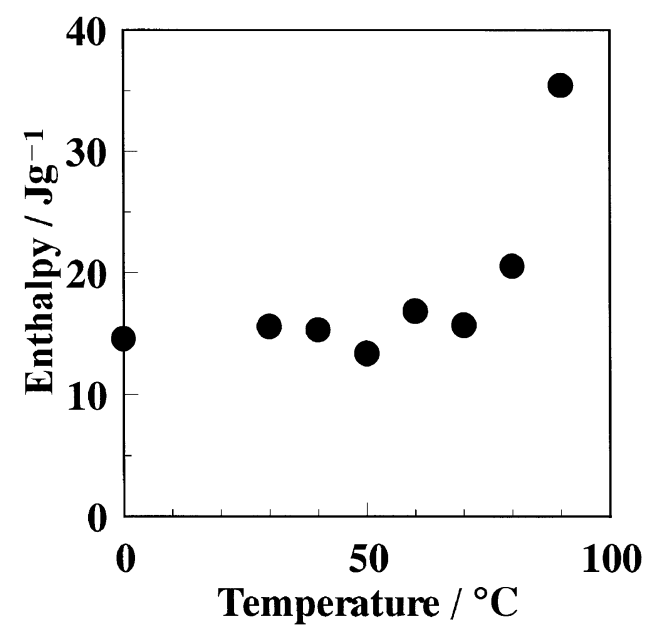

Figure 4. Melting enthalpy depending on incubated temperature.

ature as shown in Figure 3. The melting temperature of gel slightly shifted to higher temperature. Perhaps this corresponds to the growth of crystalline lamella formed at cross-linking point. For the sample incubated over $80^{\circ} \mathrm{C}$, the peak top temperature abruptly shifted to $120^{\circ} \mathrm{C}$. The crystal growth for the gel was inhibited due to entanglement of chain, however the crystallization by phase separation allowed the larger crystal size possible. In Figure 4 shows the melting enthalpy $(\Delta H)$ depending on annealing temperature. There is no large difference for the gel through all concentration, but $\Delta H$ observed for the precipitation crystal incubated at $90^{\circ} \mathrm{C}$ was more than twice compared with that for the gel. This shows that the crystallization is accelerated at high temperature, as a result the polymer chain tends to form more complete crystal. The small value of $\Delta H$ for the gel might be interpreted either by the crystallinity or the incompleteness of the crystal. Therefore high-resolution solid-state ${ }^{13} \mathrm{C}$ NMR spectroscopy was applied to make clear the molecular structure formed in

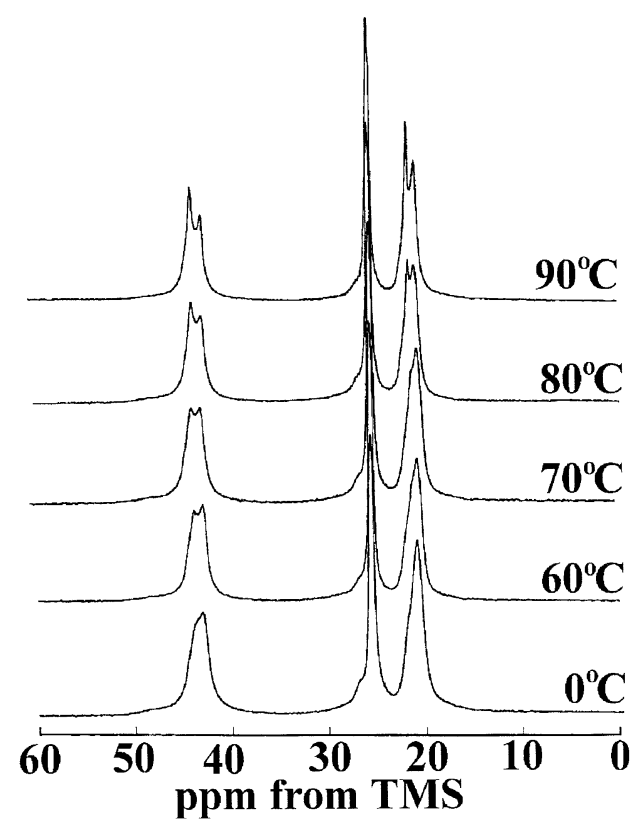

Figure 5. $\mathrm{CP} / \mathrm{MAS}{ }^{13} \mathrm{C}$ NMR spectra of iPP/o-dichlorobenzene depending on incubated temperature.

the gel.

\section{High-Resolution Solid-State ${ }^{13}$ C NMR Analysis}

For crystalline polymer, the cross-linking point is consisted of crystal so that high-resolution solid-state ${ }^{13} \mathrm{C}$ NMR can be applied to elucidate the molecular structure. We reported that the gel quenched from the homogeneous solution provides different spectral profile from other crystalline forms. In this investigation, the incubated temperatures were changed and then $\mathrm{CP} / \mathrm{MAS}{ }^{13} \mathrm{C}$ NMR spectra were measured as shown in Figure 5. Three signals due to methylene, methine, and methyl carbons were observed around $44 \mathrm{ppm}, 26 \mathrm{ppm}$, and $20 \mathrm{ppm}$, respectively. The most stable $\alpha$-form is characterized by doublet for methylene and methyl signals in CP/MAS ${ }^{13} \mathrm{C}$ NMR spectrum. The spectral profile of the precipitation crystallized over $80^{\circ} \mathrm{C}$ is identified with the $\alpha$-form, directly indicating the crystallization of the $\alpha$-form. The $\gamma$-gauche effect is well-known that the upfield shift about $5 \mathrm{ppm}$ is observed when the methylene carbon takes the relationship of gauche conformation with neighboring carbon. So the methylene signal can be used for conformational analysis. The chemical shift of methylene for the gel is almost the same as the crystal, indicating the conformational structure takes $3 / 1$ helix. With decreasing incubated temperature the intensity ratio of doublet changed. The larger the supercooling temperature was, the broader the peak profile was. Since the free induction decay (FID) is represented by exponential function in most cases, the Fourier-transformed signal has the spectral shape of Lorentz function. When two peaks are closely 


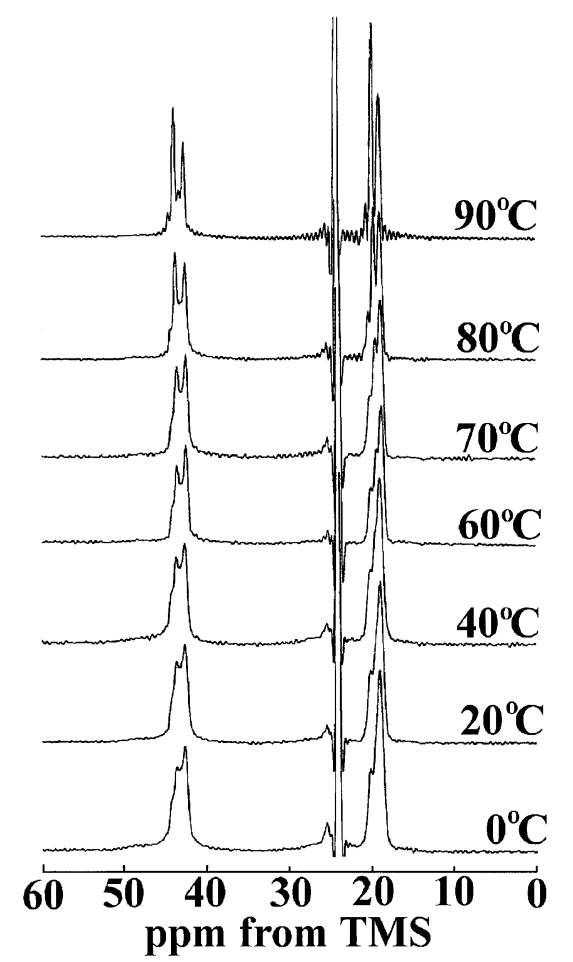

Figure 6. Lorentz-Gauss converted spectra for iPP/o-dichlorobenzene.

located, however, Lorentz function does not provide so high resolution. In order to make clear the signals, Lorentz-Gauss conversion was applied. The final peak $E(v)$ is expressed by the following equation.

$$
E(v)=\int_{-\infty}^{\infty} A(t) \exp (2 \pi i v t) \mathrm{d} t * \delta\left(v-v_{0}\right)
$$

where $v, v_{0}$, and $A(t)$ denote the frequency, the center frequency of peak and the window function, respectively. The signal is represented by the convolution (denoted by $*$ ) of Fourier transformation of $A(t)$ and $\delta$ function. When the Gauss function is adopted as window function, the narrower peak shape can be obtained. Figure 6 shows the final results converted. The methylene and methyl signals were well-resolved. However the conversion was not appropriate for methine signal as seen in Figure 6. Perhaps the decay function of methine signal cannot be represented by simple exponential function. The intensity ratio of the doublet for methylene and methyl carbons became apparently opposite compared with the $\alpha$-form. In general, CP/MAS ${ }^{13} \mathrm{C}$ NMR measurement is no quantitative method to estimate the integral intensity, because $\mathrm{CP}$ efficiency varies depending on correlation time of carbon, which directly corresponds to the molecular mobility. Especially, non-crystalline phase in the gel has the molecular mobility in the liquid. Therefore it is impossible to compare the intensity ratio between crystalline and non-crystalline phases. In this analysis, only the doublet contributed from crystalline com-

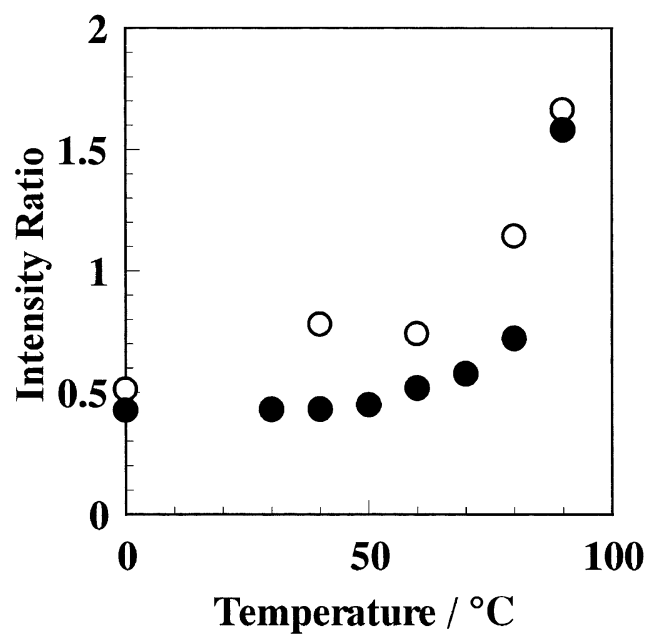

Figure 7. Intensity ratio of the doublet for methylene (O) and methyl $(\bigcirc)$ signals. The downfield peak was normalized by the upfield one.

ponent was investigated. In addition to the crystalline region, non-crystalline and intermediate phases were also included in the peak. According to the previous method, ${ }^{25}$ we carried out the computer fitting by using two crystalline, non-crystalline, and intermediate components. The intensity ratio of crystalline was compared by dividing the integral intensity of downfield peak by upfield one. The estimated values for methylene and methyl signals were plotted against annealing temperature as shown in Figure 7. The gel incubated at $0^{\circ} \mathrm{C}$ took the value of about 0.5 for both methylene and methyl signals, indicating that the downfield peak is about twice weaker than upfield one. This intensity ratio is inverse with the $\alpha$-form. According to Caldas et al., this ratio was observed for $\alpha_{1}$-form, being disordered $\alpha$-from. It was explained by the inverse cell constant of $\mathrm{a}$ and $\mathrm{b}$ axis, that is the disordered $\alpha_{1}$-form has $a=5.30 \AA$ and $b=5.17 \AA$, while the ordered $\alpha_{2}$-form $a=5.13 \AA$ and $b=5.34 \AA$. Judging from the intensity ratio in $\mathrm{CP} / \mathrm{MAS}{ }^{13} \mathrm{C}$ NMR spectrum, the gel-forming structure is regarded as disordered $\alpha$-form, corresponding to the $\alpha_{1}$-form. As described above section, $\Delta H$ of the gel was smaller than the crystal. This led to the same result to contain the incompleteness of the crystal. It is worth noting that the formation of disordered phase requires the rapid quenching process for bulk sample, but the gelation makes it possible without rapid cooling. This can be explained by the chain entanglement which allowed the crystal growth a void. Below $70^{\circ} \mathrm{C}$, the intensity ratio gradually increased with annealing temperature. Since the gel structure of iPP contains large amount of defects in the crystalline cell, the crystalline order is slightly improved by incubating at high temperature. The downfield peak of doublet increased in intensity over $80^{\circ} \mathrm{C}$, being the boundary 


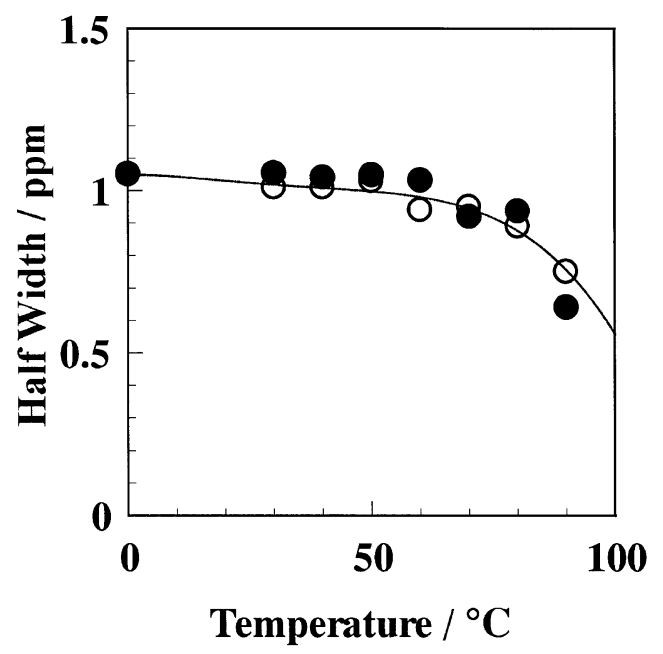

Figure 8. Half width for methylene $(\bullet)$ and methyl $(\bigcirc)$ signals depending on incubated temperature.

temperature for gelation and crystallization. This corresponds to the crystallization to the high ordered $\alpha$-form. Figure 8 shows the half width for two crystalline components as a function of annealing temperature. With increasing temperature, the half width became slightly narrow for the gel, corresponding to the improvement of crystalline order. The peak shape was much narrower over $80^{\circ} \mathrm{C}$. This corresponds to the formation of more complete crystal like single crystal.

\section{CONCLUSION}

The molecular structure and morphology grown from iPP/o-dichlorobenzene kept at constant temperature were investigated by DSC and high-resolution solidstate ${ }^{13} \mathrm{C}$ NMR. The homogeneous gel was formed below $70^{\circ} \mathrm{C}$, while the phase separation occurred over $80^{\circ} \mathrm{C}$ and the crystal grew from the solution. The $\mathrm{CP} / \mathrm{MAS}{ }^{13} \mathrm{C}$ NMR spectrum obtained through crystallization over $80^{\circ} \mathrm{C}$ was identified with high ordered $\alpha$ form, because the characteristic doublets for methylene and methyl signals were observed. The gel provided very broad peaks in the $\mathrm{CP} / \mathrm{MAS}{ }^{13} \mathrm{C}$ NMR spectrum. In order to make clear the spectral shape, the LorentzGauss conversion was computationally calculated. The methylene and methyl signals consisted of two crystalline components. The doublet located at the same chemical shift as the $\alpha$-form, but the intensity ratio was different. This indicates that the molecular conformation takes the $3 / 1$ helix, having the same conformation as the $\alpha$-form, but the crystalline order is incomplete. This result was also led from DSC measurement. The gel formed below $70^{\circ} \mathrm{C}$ took lower $T_{\mathrm{m}}$ and smaller $\Delta H$ than the crystal. This corresponds that the crystalline order is not so high in the gel. It was reported that the disordered phase is formed through rapid quenching process, but the gelation is regarded as crystallization from the high concentration solution so that the disordered crystal is formed due to chain entanglement.

Acknowledgment. This work was financially supported by High Tech Research Center at Ryukoku University.

\section{REFERENCES}

1. G. Natta, P. Pino, P. Corradini, F. Danuss, E. Mantica, G. Mazzanii, and G. Moriglio, J. Am. Chem. Soc., 77, 1708 (1955).

2. C. C. Hsu and P. H. Geil, J. Polym. Sci., Polym. Phys. Ed., 24, 2379 (1986).

3. D. T. Grubb and D. Y. Yoon, Polym. Commun., 27, 84 (1986).

4. P. Corradini, V. Petraccone, C. De Rosa, and G. Guerra, Macromolecules, 19, 2699 (1986).

5. P. Corradini, G. Guerra, and V. Petraccone, Polym. Commun., 30, 281 (1989).

6. R. J. Yan, G. Li, and B. Jiang, Macromolecular Sci. Phys., B32, 15 (1993).

7. M. A. Gomez, H. Tanaka, and A. E. Tonelli, Polymer, 28, 2227 (1987).

8. S. V. Meille, D. R. Ferro, S. Brukner, A. L. Lovinger, and F. J. Padden, Macromolecules, 27, 2615 (1994).

9. S. Brukner and S. V. Meille, Nature, 340, 455 (1989).

10. S. V. Meille, S. Brukner, and W. Porzio, Macromolecules, 23, 4114 (1990).

11. S. Bruckner, S. V. Meille, P. Sozzani, and G. Torri, Makromol. Chem., Rapid Commun., 11, 55 (1990).

12. R. A. Campbell, P. J. Phillips, and J. S. Lin, Polymer, 34, 4809 (1993).

13. R. L. Miller, Polymer, 1, 135 (1960).

14. R. Hosemann and W. Wilke, Makromol. Chem., 118, 230 (1968).

15. J. A. Gailey and R. H. Ralston, SPE Trans, 4, 29 (1964).

16. V. Caldas, G. R. Brown, R. S. Nohr, J. G. MacDonald, and L. E. Raboin, Polymer, 35, 899 (1994).

17. V. Caldas, F. G. Morin, and G. R. Brown, Magn. Reson. Chem., 32, 72 (1994).

18. V. Caldas, G. R. Brown, R. S. Nohr, and J. G. MacDonald, J. Polym. Sci., Part B: Polym. Phys., 34, 2085 (1996).

19. Z. G. Wang, B. H. Hsiao, S. Srinivas, G. M. Brown, A. H. Tsou, S. Z. D. Cheng, and R. S. Stein, Polymer, 42, 7561 (2001).

20. A. Bunn, M. E. A. Cudby, R. K. Harris, K. J. Packer, and B. J. Say, Polymer, 23, 694 (1982).

21. S. Saito, Y. Moteki, M. Nakagawa, F. Horii, and R. Kitamaru, Macromolecules, 23, 3256 (1990).

22. M. Kobayashi, K. Tsumura, and H. Tadokoro, J. Polym. Sci., $A-2$, 6, 1493 (1968).

23. T. Nakaoki, H. Hayashi, and R. Kitamaru, Polymer, 37, 4833 (1996).

24. T. Nakaoki, Y. Ohira, and F. Horii, Polymer, 42, 4555 (2001).

25. T. Nakaoki, H. Shuto, H. Hayashi, and R. Kitamaru, Polymer, 39, 3905 (1998).

26. H. Matsuda, T. Inoue, M. Okabe, and T. Ukaji, Polym. J., 19, 323 (1987). 